\title{
Aplicación del Lean Manufacturing (5s y Kaizen) para el incremento de la productividad en el área de producción de adhesivos acuosos de una empresa manufacturera
}

EDITH LuZ VARGAS CRISÓSTOMO José William CAMERo JimÉNEZ ${ }^{2}$

\begin{abstract}
RESUMEN
La presente investigación se desarrolla en una empresa manufacturera que en los últimos cuatro años ha estado presentando un problema de baja productividad en el área de producción de adhesivos acuosos, cuyos valores de productividad son inferiores a $5 \mathrm{Kg} / \mathrm{h}-\mathrm{h}$, que es el valor esperado. Por consiguiente, en el presente estudio se consideró necesario aplicar una estrategia de mejora basada en la metodología de Lean Manufacturing, por lo que se seleccionó la metodología Kaizen y las 5S. La aplicación se llevó a cabo en etapas que consistieron, primero, en un diagnóstico situacional, seguido del diseño, la implementación y la evaluación de los resultados; estas etapas se desarrollaron en 7 meses, de enero a julio del 2019. Al culminar la aplicación de la metodología del Lean Manufacturing, se evaluaron los resultados de la productividad y se obtuvo un valor promedio de $5.58 \mathrm{Kg} / \mathrm{h}$-h. Cabe mencionar que, en el año 2018, antes de la aplicación del Lean Manufacturing, se tenía un valor promedio de productividad de $4.37 \mathrm{Kg} / \mathrm{h}-\mathrm{h}$.

Palabras clave: Lean Manufacturing; Productividad; $5 S$ y Kaizen.
\end{abstract}

\section{INTRODUCCIÓN}

Los adhesivos acuosos son utilizados ampliamente como aditivos o productos intermedios en diferentes industrias, tales como la maderera, papelera, embotelladora, entre otras. Nuevas empresas aparecen en el rubro por causa de un mercado cada vez más competitivo; por tal razón, para que las empresas puedan subsistir como industria, es necesario aplicar estrategias que las ayuden a mantenerse competitivas y posicionarse en el mercado nacional, donde no solo la calidad es determinante, sino también la mejora del sistema de producción, el cual está basado en la eliminación de actividades que no aportan valor al proceso ni al cliente.

La empresa manufacturera en estudio, que se dedica a la fabricación de adhesivos acuosos, requiere cumplir con determinados objetivos y metas, así como tomar decisiones acertadas que garanticen la permanencia de la empresa en el mercado y una alta rentabilidad que repercuta en beneficios para los trabajadores y sus accionistas; sin embargo, en los últimos 4 años, la productividad ha sido baja. Para revertir esta situación, se requiere aplicar nuevas metodologías basadas en la mejora de procesos como, por ejemplo, el Lean Manufacturing, el cual permite incrementar la productividad por medio de la eliminación de desperdicios.

La metodología de Lean Manufacturing, se define como un modelo de gestión de excelencia y mejora continua que consiste en la eliminación de los desperdicios que no agregan valor al producto y está conformada por diferentes herramientas. Para la presente investigación se aplicaron las herramientas $5 \mathrm{~S}$ y Kaizen, para lo cual, se inició con la revisión bibliográfica del Lean Manufacturing, se realizó el diseño, se continuó con la implementación y se finalizó con la evaluación de los resultados con métodos estadísticos.

1 Ingeniera química por la Universidad Nacional del Centro del Perú (Junín, Perú). Actualmente, trabaja como consultora independiente (Lima, Perú).

ORCID: https://orcid.org/0000-0001-9637-5365

Autor de correspondencia: edith luz91@hotmail.com

2 Estadístico por la Universidad Nacional de Ingeniería (Lima, Perú). Actualmente, es docente de la Escuela Profesional de Estadística de la Universidad Nacional de Ingeniería (Lima, Perú). ORCID: https://orcid.org/0000-0003-0517-3237

E-mail: jcameroj@uni.edu.pe 
La filosofía Lean se considera novedosa y relevante dentro de las empresas, pues permite optimizar los procesos de producción, donde se busca eliminar las actividades que no aportan valor en las diferentes etapas de las operaciones y que no proveen beneficios a la empresa. Esta eliminación se aplica desde la iniciación hasta el cierre de la producción, lo que mejora la economía y ahorro de recursos.

El aporte al conocimiento del estudio es la orientación principal de optimizar procesos y mejorar la productividad por medio de la reducción de tiempos muertos, lo que se traduce en incrementar ganancias, mejorar la calidad del producto y generar una cultura de cambio de pensamiento en las personas, para lograr eficacia del proceso de producción, es decir, producir más con menos recursos y esfuerzo.

Para aplicar los resultados obtenidos a otras organizaciones, se deben evaluar todos los procesos de producción y servicios e identificar dónde se requiere aplicar esta metodología, fundamentalmente para dar soluciones a los problemas a través de la mejora continua. La implementación del Lean Manufacturing se aplica mediante seis pasos: Formación de un equipo Lean, capacitación, diagnóstico (identificación de desperdicios), aplicación de la metodología Lean, análisis de resultados y elaboración de planes de acción enfocados a la reducción de tiempo de procesos para lograr la reducción de costos por mala calidad, reducción de defectos, mejora de rentabilidad de la organización, entre otros.

El objetivo general del estudio es "aplicar el Lean Manufacturing (5S y Kaizen) para el incremento de la productividad en el área de producción de adhesivos acuosos de una empresa manufacturera". De este, se desprenden los objetivos específicos "analizar la situación actual de la productividad del área de producción de adhesivos acuosos de una empresa manufacturera", "aplicar la metodología del Lean Manufacturing en el área de producción de adhesivos acuosos de una empresa manufacturera" y "evaluar la productividad después de aplicar la metodología del Lean Manufacturing en el área de producción de adhesivos acuosos de una empresa manufacturera".

\section{JUSTIFICACIÓN}

El sector de la fabricación de los adhesivos es cada vez más grande, y tendría un avance significativo en los próximos años, puesto que ofrece beneficios como, por ejemplo, rendimientos equivalentes y, en ocasiones, mejores a los de otros productos usados en ensambladura y sellado de superficies, con ventajas en cuanto a reducción de costos. Asimismo, los adhesivos son usados como productos intermedios e insumos en una variedad de productos industriales, entre los que tenemos: adhesivo de etiquetado, adhesivo de laminación (papel higiénico), adhesivo de cartón, entre otros; por esto, la fabricación de adhesivos acuosos se convierte en un negocio apreciable.

En la actualidad, la cantidad de empresas fabricantes de adhesivos acuosos, que ofrecen productos y servicios de calidad, ha incrementado, lo que resulta en un mercado competitivo. Por lo expuesto, la presente investigación se enfoca en aplicar una estrategia de mejora mediante el Lean Manufacturing (5S y KAIZEN) y en evaluar el impacto en el incremento de la productividad.

\section{HIPÓTESIS}

- $\mathrm{H}_{0}=$ La productividad del 2019 es igual a la productividad del 2018.

- $\mathrm{H}_{\mathrm{a}}=$ La productividad del 2019 es mayor a la productividad del 2018.

\section{MARCO TEÓRICO}

\section{Adhesivos}

Se define adhesivo como la aplicación de todas las fuerzas que se oponen a la división de las moléculas de diferentes contexturas; por consiguiente, la adherencia es la resistencia que se opone a la separación de los cuerpos (Bilurbina y Liesa, 1990).

\section{Adhesivos acuosos}

Los adhesivos de base acuosa son disoluciones de polímeros (de naturaleza vegetal o sintético) disueltos en agua y se caracterizan por tener una velocidad de fraguado menor que los adhesivos en base solvente, ya que el agua tarda más en evaporarse que los adhesivos orgánicos (Gonzáles, 2014).

Los adhesivos en base acuosa se presentan como una de las mejores alternativas a los que utilizan solventes orgánicos, que son muchos más tóxicos y contaminantes (Gonzáles, 2014).

\section{Lean Manufacturing}

Se entiende por Lean Manufacturing (producción esbelta), la búsqueda de la mejora del sistema de fabricación por medio de la eliminación o disminución de los desperdicios o muda, que son todas las acciones que no contribuyen valor al producto ni al servicio, que son causadas por actividades que consumen recursos sin crear valor alguno y que el 
cliente no está dispuesto a pagar (Womack y Jones, 1996).

La metodología Lean Manufacturing busca reducir al mínimo los desperdicios de forma permanente para maximizar las etapas del proceso, centrándose en la reducción de costos y en la minimización de desperdicios y de materiales en la cadena de suministro (Vinodh y Dino, 2012), lo que se puede resumir en los siguientes objetivos:

- Aprovechar el uso de los activos organizacionales;

- Mejorar la satisfacción del cliente externo e interno;

- Mejorar las competencias del personal tanto en conocimientos como en habilidades;

- Eliminar los desperdicios (actividades que no agregan valor) en el proceso de fabricación.

\section{Pilares del Lean Manufacturing}

\section{- El Control total de la calidad}

El control total de calidad es la garantía de calidad desde las primeras etapas del desarrollo de un producto. Su función es asegurar que los productos o servicios cumplan con los requisitos de calidad, lo que se logra con la participación de todos los colaboradores de todos los procesos; así, se da origen al control de calidad en toda la organización, que es la cualidad más importarte del control de calidad japonés (Ishikawa, 1997).

El control de calidad se aplica primordialmente a una organización para conocer las especificaciones establecidas por la ingeniería del producto y para proporcionar asistencia al departamento de fabricación de modo que la producción alcance estas especificaciones. Como tal, su función consiste en la recolección y análisis de grandes cantidades de datos que después se presentan a diferentes departamentos para la evaluación de dichos resultados.

\section{- Mejora continua}

La mejora continua es el objetivo permanente de las organizaciones, pues se centra en la resolución de problemas. El ciclo PHVA, hoy en día considerado una herramienta de mejora esencial, fue presentado por Deming, quien lo describió en 4 pasos (Socconini, 2018):
- Planificar: Un cambio o test con objetivo de mejorar.

- Hacer: Llevar a cabo el cambio o test, preferiblemente a pequeña escala.

- Verificar: Estudiar los resultados. ¿Qué hemos aprendido?

- Actuar: Adoptar el cambio, abandonarlo, o recorrer el ciclo de nuevo, posiblemente bajo diferentes condiciones ambientales.

\section{- Just In Time (JIT)}

Taiichi Ohno, primer vicepresidente de Corporation Toyota Motor, desarrolló el sistema de producción Just in time buscando la reducción de costos a través de la eliminación de los desperdicios. Ohno tomó conceptos desarrollados por Henry Ford y Walter Shewhart entre los años de 1920 y 1930; con base en estos, desarrolló una filosofía de excelencia en manufactura que superó a todas las anteriores. (Rajadell y Sánchez, 2010).

\section{- Productividad}

Es la correlación que existe entre la producción y el uso óptimo de los recursos financieros, materiales y humanos para lograr los objetivos organizacionales, incrementar la calidad de los servicios y bienes producidos y buscar el desarrollo de los colaboradores (Rodríguez, 1999).

\section{- Eficacia}

Es la aptitud que desarrolla una organización para incrementar ganancias por diferentes medios, incluyendo la capacidad para conseguir el efecto deseado y el manejo de los inputs y outputs del entorno, como la política u otros (Fernández y Sánchez, 1997).

\section{- Eficiencia}

La eficiencia se alcanza cuando los objetivos planteados se logran con la mínima cantidad de inputs. Por ejemplo, podemos incrementar la eficiencia en una jornada de trabajo eliminando las pausas y reduciendo el tiempo de almuerzo, con lo que la producción aumentaría; sin embargo, es posible que el número de errores aumente debido a la fatiga del empleado, es decir, se habrá conseguido un sistema de producción eficiente de productos ineficientes. Por ello, se propone que la productividad sea una relación entre la eficacia y eficiencia conseguida, monitoreando los costos de 
manufactura en que la eficacia es hacer lo adecuado y la eficiencia es la manera de hacer bien las cosas (Huertas y Domínguez, 2015).

\section{- Las $5 \mathrm{~S}$ :}

Las $5 \mathrm{~S}$ se describen como una metodología de mejora que se originó en Japón. Esta consiste en la clasificación, el orden y limpieza del área, la estandarización de procesos y la disciplina del personal, de modo que sean hábitos en las áreas de trabajo y se logre una cultura de mejora laboral. Este método se puede desarrollar en cualquier rubro y en todas las áreas de trabajo de una empresa (Barcia y Hidalgo, 2013).

o Seiri: Seleccionar. Consiste en determinar y diferenciar lo que realmente es necesario o imprescindible para nuestro lugar de trabajo. Se retienen los elementos necesarios, para después clasificarlos y eliminar lo innecesario. Se reducen los elementos del puesto de trabajo, de modo que solo queden los imprescindibles para completar los procesos y tareas del área con la máxima eficacia y eficiencia (Aldavert, Vidal, y Lorente, 2017).

o Seiton: Organizar. Consiste en colocar lo necesario en un lugar fácilmente accesible, colocar las cosas útiles por orden según criterios de Seguridad/Calidad/Eficacia.
- Seguridad: Que no se puedan caer, que no se puedan mover, que no estorben.

- Calidad: Que no se golpeen, oxiden, mezclen ni deterioren.

- Eficacia: Que se minimice el tiempo perdido (Vargas, 2004).

o Seiso: Limpieza. Pretende incentivar la actitud de limpieza del sitio de trabajo y la conservación de la clasificación y el orden de los elementos. El proceso de implementación se debe apoyar en un fuerte programa de entrenamiento y suministro de los elementos necesarios para su realización, como también del tiempo requerido para su ejecución (Vargas, 2004).

o Seiketsu: Estandarizar. Consiste en lograr mantener constantemente el orden, limpieza e higiene del sitio de trabajo. Se logra limpiando con la regularidad establecida, manteniendo todo en su sitio y en orden, estableciendo procedimientos y planes para mantener el orden y la limpieza (Vargas, 2004).

o Shitsuke: Disciplina. Consiste en lograr tener el hábito de aplicar las $5 \mathrm{~S}$ en el área de trabajo, respetando y haciendo respetar las normas del área de trabajo, usando siempre los implementos de protección y manteniendo el aseo y

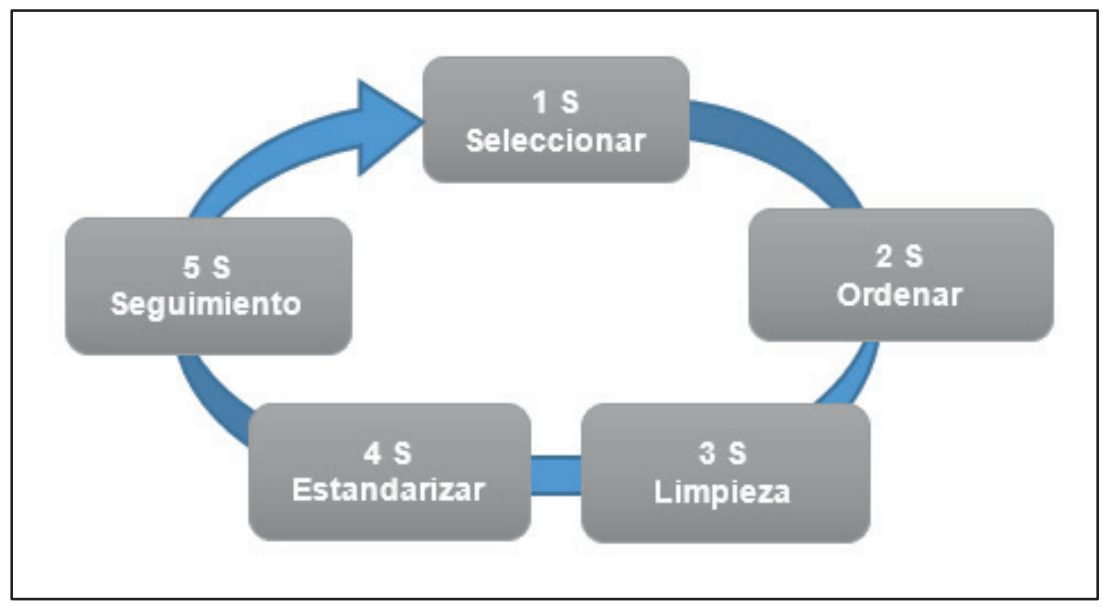

Figura 1. Las 5 S.

Fuente: Elaboración propia. 
limpieza, de modo que estos detalles se conviertan en hábitos diarios y frecuentes (Vargas, 2004).

\section{- Kaizen:}

La palabra Kaizen significa "mejorar". Al aplicarla en las organizaciones como una mejora continua en la forma de hacer las cosas, genera un impacto en el área de trabajo, así como en el entorno personal, familiar y social.

Al desarrollar una cultura de mejora continua, que incluye a todos los trabajadores, se lleva a cabo un esfuerzo complementario hacia el mejoramiento del desempeño a nivel de organización en todos los niveles, dirigido hacia la satisfacción de metas funcionales y transversales como la calidad, costos y potenciales humanos, con el fin de conseguir una mayor satisfacción del cliente (Gil, Monzon, Celma, y Giner 1996).

La aplicación del Kaizen comprende diez principios básicos que resultaron de variadas aplicaciones en distintas empresas japonesas. Gracias a estas aplicaciones se supo que la capacitación y el desarrollo de competencia de los colaboradores son los que deben generar el desarrollo de una cultura Kaizen, en la que se busca interiorizar los principios y aplicarlos de manera natural.

Según Atehortua (2010), los principios Kaizen, son:

1. Concentrarse en los requerimientos de los clientes.

2. Realizar pequeñas mejoras constantemente.

3. Identificar, reconocer y analizar abiertamente los problemas.

4. Fomentar la apertura a la comunicación.

5. Conformar equipos de trabajo "Kaizen".

6. Desarrollar los proyectos Kaizen a través de personas multifuncionales.

7. Mantener los procesos con buenas relaciones.

8. Fomentar la autodisciplina mediante reconocimientos.
9. Mantener la comunicación con los empleados.

10. Desarrollar competencias en todos los empleados.

\section{METODOLOGÍA}

\section{Análisis de la productividad}

Para realizar el análisis de la productividad, se toman los valores de los últimos 5 años (Tabla 1) y se observó que solo en el año 2014 se ha superado el valor de 5.00 kg/ h-h, que es el valor esperado por la empresa manufacturera.

Tabla 1. Resultados de Productividad por año.

\begin{tabular}{cc}
\hline Año & $\begin{array}{c}\text { Productividad } \\
\mathbf{k g} / \mathbf{h}-\mathbf{h}\end{array}$ \\
\hline 2014 & 5.23 \\
2015 & 4.75 \\
2016 & 4.60 \\
2017 & 4.87 \\
2018 & 4.37 \\
\hline
\end{tabular}

Fuente: Elaboración propia.

\section{Determinación de la productividad}

Para la determinación de la productividad en la empresa manufacturera, se realiza mediante la siguiente fórmula:

$$
\text { Productividad }=\frac{\text { Producción del mes }(\mathrm{Kg})}{\text { Total horas }- \text { hombre }(\boldsymbol{h}-\boldsymbol{h})}
$$

Producción en planta adhesivos acuosos

$=\overline{\text { Personal de producción }+ \text { personal de producción administrativo }}$

La metodología para la aplicación del Lean Manufacturing $5 \mathrm{~S}$ y Kaizen, se visualiza en la figura 2.

\section{Selección del equipo de trabajo}

Se selecciona al personal de producción y personal del área de mejora continua para dirigir y dar soporte en las actividades de aplicación de las herramientas de Lean Manufacturing (5 S y Kaizen).

\section{Capacitación}

Se capacita al equipo de trabajo seleccionado en los temas de Lean Manufacturing y las etapas de su aplicación; la capacitación está a cargo de los instructores del Instituto Lean Six Sigma de México. 


\section{Diagnóstico de las $5 \mathrm{~S}$}

Se realiza mediante una evaluación de la situación actual de las $5 \mathrm{~S}$ en el área de producción de adhesivos acuososos con la ayuda de un formato de check list para luego procesar los datos. Las imágenes de la situación actual se observan en la Figura 3.

\section{Aplicación de las $5 \mathrm{~S}$}

La aplicación consistió en seguir los lineamientos del "Manual de Implementación de las 5S", otorgado por el área de Mejora Continua, el cual se resume en los siguientes pasos:

a. Elaboración de Lay out (Plano 5S), delimitar las zonas o áreas a implementar.

b. Respecto a la 1S: Se crea la lista de necesarios y se retira lo innecesario. c. Respecto a la 2S: Se ubican todos los necesarios, entre los que se encuentran los equipos, máquinas, muebles, herramientas, materiales, entre otros, y se rotulan.

d. Respecto a la 3S: Se mantienen limpio todos los necesarios, se implementa el plan de limpieza y se realiza un seguimiento para su cumplimiento.

e. Respecto a la 4S: Se verifica que el etiquetado, rotulado y demarcado cumpla con las reglas de estandarización indicado en el Manual de Implementación de $5 \mathrm{~S}$.

f. Respecto a la 5S: Se realizan auditorías de 5 $S$ y se designa un responsable dentro del área para levantar las observaciones.

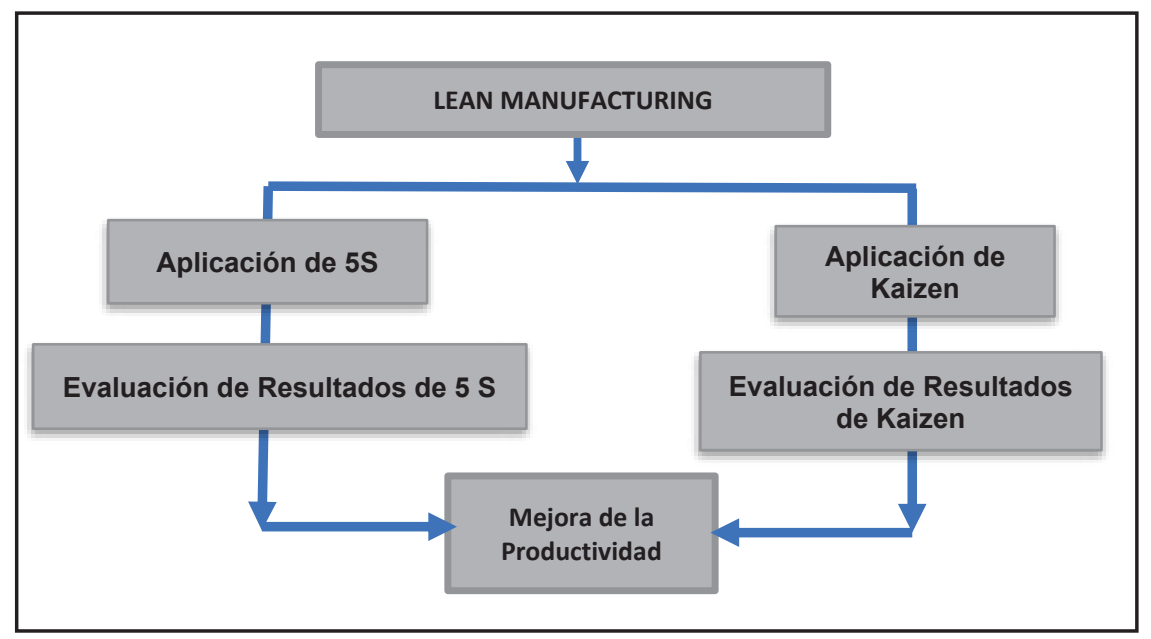

Figura 2. Implementación de la metodología Lean Manufacturing. Fuente: Elaboración propia.

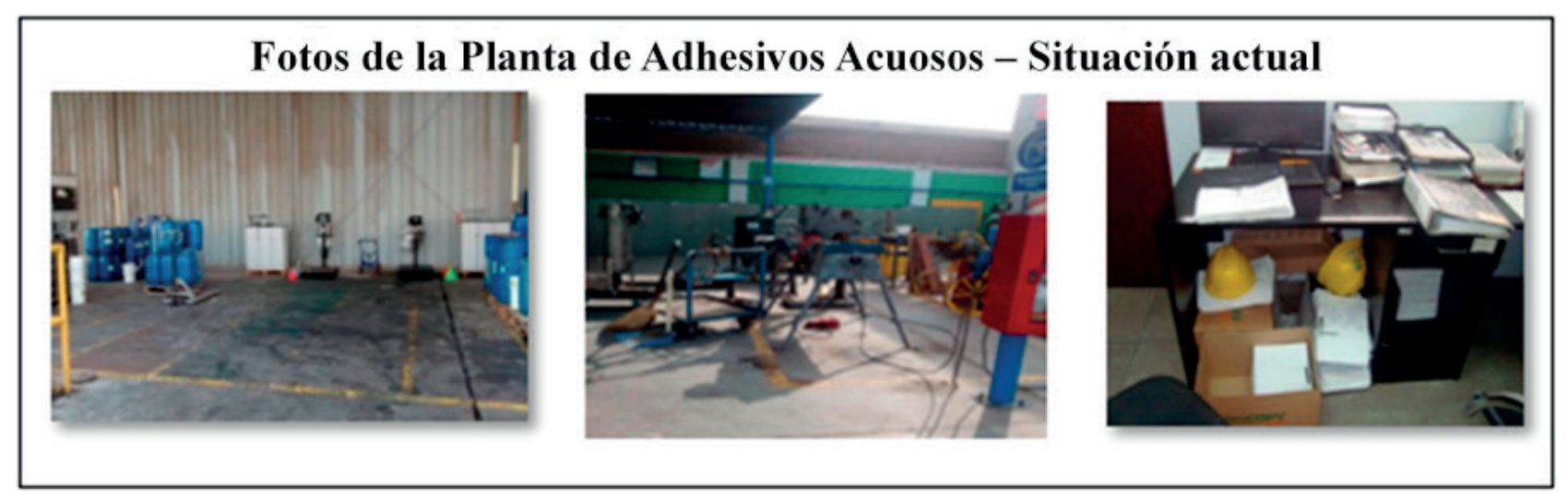

Figura 3. Situación actual de la Planta de Adhsesivos Acuosos.

Fuente: Elaboración propia. 


\section{Aplicación del Kaizen}

Con la implementación de un Kaizen, se realizan cambios que permiten mejorar el proceso de producción del producto de mayor venta. La implementación comprende las siguientes etapas.

a. Se identifica el problema en el proceso de producción de adhesivos acuosos, el cual consiste en el tiempo excesivo de proceso de fabricación del producto de mayor venta.

b. Se describe el proceso de fabricación del producto, que involucra 10 etapas en el proceso productivo.

c. Se realiza el análisis de causas usando la técnica lluvia de ideas y Pareto. La causa raíz es el tiempo de enfriamiento excesivo del reactor usado en el proceso de fabricación por falta de mantenimiento (presenta exceso de sarro). d. Se elabora un plan de acción, que incluye actividades de mantenimiento, junto al área de mantenimiento de equipos usados en el proceso productivo.

e. Se realiza el seguimiento a las actividades implementadas y se evalua su efectividad.

\section{Resultados de las $5 \mathrm{~S}$}

a. Los resultados del diagnóstico de 5S, realizado antes del inicio de la aplicación del Lean Manufacturing, en el mes de enero del 2018se muestran en la Tabla 2 y en las imágenes en la Figura 4.

b. Los resultados de la auditoría $5 \mathrm{~S}$, realizada después de aplicar la metodología de Lean Manufacturing, en los meses de abril a julio del 2019, se muestran en la Tabla 3 y en las imágenes en la Figura 5.

Tabla 2. Resultados del diagnóstico de 5S.

\begin{tabular}{|l|l|c|}
\hline \multicolumn{2}{|c|}{ Pilares de 5 S } & Diagnóstico de 5 S \\
\hline 1 S & Seleccionar & 3.0 \\
\hline 2 S & Ordenar & 2.5 \\
\hline 3 S & Limpieza & 2.7 \\
\hline 4 S & Estandarización & 2.2 \\
\hline 5 S & Seguimiento & 3.5 \\
\hline Promedio & 2.8 \\
\hline
\end{tabular}

Fuente: Elaboración propia.

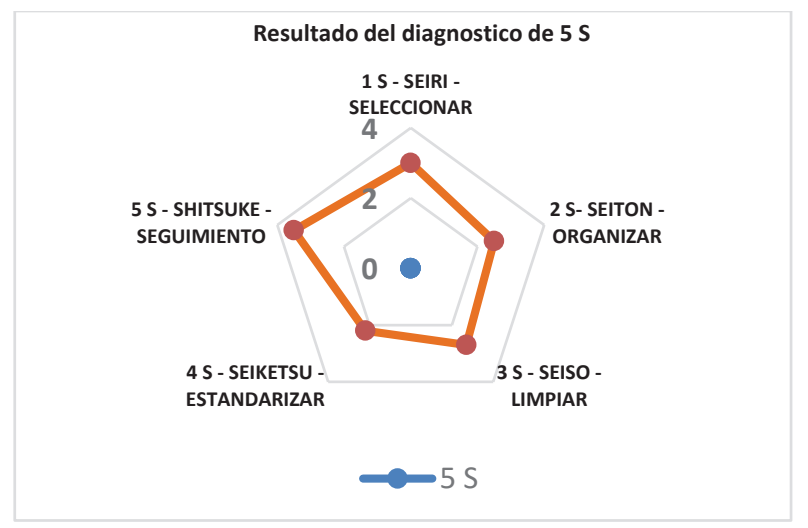

Figura 4. Resultados de diagnóstico de $5 \mathrm{~S}$.

Fuente: Elaboración propia.

Tabla 3. Resultados de la auditoría de $5 \mathrm{~S}$.

\begin{tabular}{|c|c|c|}
\hline \multicolumn{2}{|c|}{ Meses del 2019 } & Puntaje Auditoría de 5 S \\
\hline 1 & Abril & 4.0 \\
\hline 2 & Mayo & 4.0 \\
\hline 3 & Junio & 4.5 \\
\hline 4 & Julio & 4.5 \\
\hline
\end{tabular}

Fuente: Elaboración propia. 


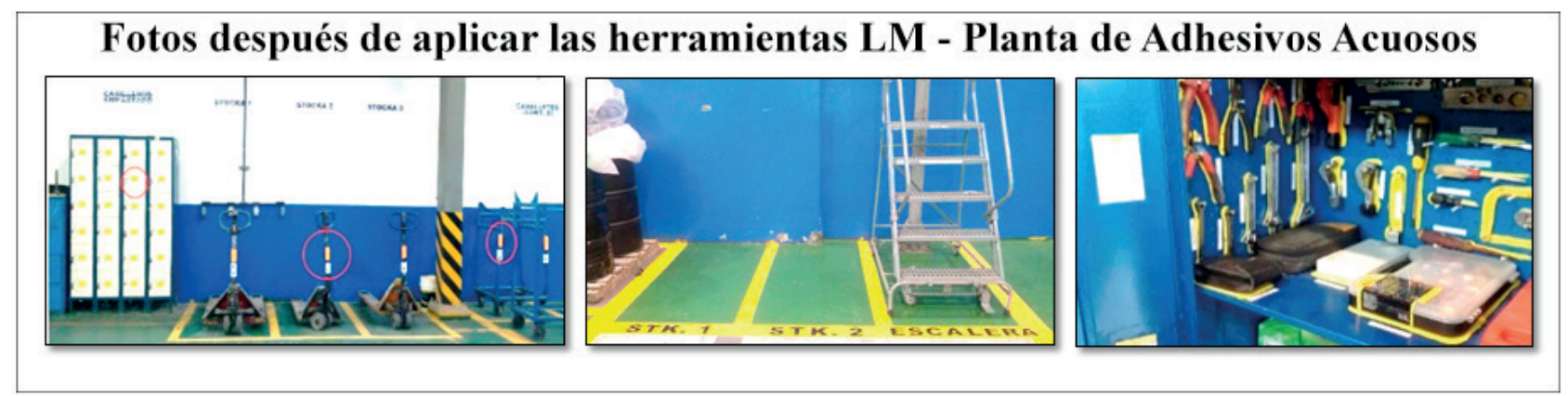

Figura 5. Resultados del diagnóstico de $5 \mathrm{~S}$.

Fuente: Elaboración propia.

\section{Resultados obtenidos del Kaizen}

En la Tabla 4 se muestran los resultados de aplicar Kaizen al problema encontrado (tiempo de fabricación extenso del producto con mayor movimiento de ventas); se observa que la aplicación de Kaizen redujo los tiempos de fabricación en hasta 2 horas y 23 minutos.

\section{Datos de la productividad}

En la Tabla 5 se muestran los datos de productividad obtenidos en el área de producción de adhesivos acuosos para el 2018 (antes de aplicar la metodología del Lean Manufacturing) y los datos de la productividad del 2019 (después de aplicar la metodología Lean Manufacturing) (Figura 6).

\section{PRUEBA DE HIPÓTESIS}

De acuerdo con el planteamiento de la hipótesis y los datos mostrados en la Tabla 5, se realiza la evaluación mediante el programa Minitab a un nivel de significancia de $\alpha=0.05$; se realiza la prueba de normalidad $t$ de Student y la gráfica de cajas a fin de demostrar si se acepta o se rechaza la hipótesis nula.

\section{Prueba estadística de normalidad}

Para el desarrollo de la prueba estadística de normalidad, se utiliza la prueba Anderson-Darling para los datos de la productividad del año 2018 y 2019 (Figura 7 y 8 ), los mismos fue fueron aceptados por tener un $p$-valor $>\alpha$ al $95 \%$ de confianza.

\section{Prueba $t$ de Student}

Después de determinar que los datos tienen una distribución normal, se realiza la prueba $t$ de Student para ambas muestras. El resultado se muestra en la Tabla 6 , en el que se obtuvo que $p$-valor $<\alpha$
$(0.00<0.05)$, por lo cual se rechaza la hipótesis nula y se acepta la hipótesis alterna. Así, se concluye que la productividad del 2019 es mayor a la productividad del 2018 puesto que se aplicó la metodología del Lean Manufacturing (Tabla 6).

\section{Prueba de cajas}

Mediante la gráfica de cajas, se puede apreciar visualmente el incremento de la productividad, como se muestra en la Figura 9.

\section{DISCUSIÓN}

Al aplicar las metodologías del Lean Manufacturing (5S y Kaizen), se mejoran los resultados de la productividad del área de producción de adhesivos acuosos de una compañía manufacturera, los mismos que se corroboraron mediante la prueba de hipótesis. Así, quedó demostrado que la productividad incrementa al aplicar el Lean Manufacturing.

\section{CONCLUSIONES}

Se realizó un estudio de línea base o diagnóstico de $5 S$ antes de la aplicación del Lean Manufacturing, donde se obtuvo un valor inicial promedio de 2.8. Al culminar la aplicación de las $5 \mathrm{~S}$, se obtuvo como resultado de auditoría el valor promedio de 4.03 al reducir los tiempos innecesarios por búsqueda de materiales y por transporte del personal, además de mejorar la organización y la limpieza del área de producción de adhesivos acuosos.

Al aplicar la metodología Kaizen, se redujo el tiempo de proceso de fabricación del producto con mayor índice de ventas en el área de producción de adhesivos acuosos. Antes de aplicar Kaizen, se tenía un tiempo de 20:15 horas y, después de la aplicación, se obtuvo un tiempo de fabricación de 17:09 horas, es decir, se disminuyó el tiempo de fabricación en 3 horas y 6 minutos. 
Tabla 4. Resultados al aplicar Kaizen.

\begin{tabular}{|c|c|c|}
\hline Herramientas Kaizen & $\begin{array}{c}\text { Meses } \\
\mathbf{( 2 0 1 9 )}\end{array}$ & $\begin{array}{c}\text { Tiempo de Fabricación } \\
\text { (Horas) }\end{array}$ \\
\hline \multirow{3}{*}{ Antes de aplicar Kaizen } & Enero & $20: 15: 00$ \\
\cline { 2 - 3 } & Febrero & $20: 04: 00$ \\
\cline { 2 - 3 } & Marzo & $20: 10: 00$ \\
\hline \multirow{3}{*}{ Después de aplicar Kaizen } & Abril & $18: 19: 00$ \\
\cline { 2 - 3 } & Mayo & $18: 46: 00$ \\
\cline { 2 - 3 } & Junio & $17: 26: 00$ \\
\cline { 2 - 3 } & Julio & $17: 09: 00$ \\
\hline
\end{tabular}

Fuente: Elaboración propia.

Tabla 5. Datos de la productividad 2018 y 2019.

\begin{tabular}{|c|c|c|c|}
\hline Fecha & Semana & $\begin{array}{c}\text { Productividad } 2018 \\
\text { (kg/ h-h) }\end{array}$ & $\begin{array}{c}\text { Productividad } 2019 \\
\text { (kg/ h-h) }\end{array}$ \\
\hline \multirow{4}{*}{ Enero } & Sem 1 & 4.93 & 5.20 \\
\hline & Sem 2 & 4.75 & 5.35 \\
\hline & Sem 3 & 4.60 & 5.44 \\
\hline & Sem 4 & 4.25 & 5.24 \\
\hline \multirow{4}{*}{ Febrero } & Sem 5 & 4.42 & 5.54 \\
\hline & Sem 6 & 4.23 & 5.66 \\
\hline & Sem 7 & 4.60 & 5.48 \\
\hline & Sem 8 & 4.83 & 5.51 \\
\hline \multirow{4}{*}{ Marzo } & Sem 9 & 4.62 & 5.72 \\
\hline & Sem 10 & 3.93 & 5.61 \\
\hline & Sem 11 & 3.20 & 5.66 \\
\hline & Sem 12 & 4.30 & 5.75 \\
\hline \multirow{4}{*}{ Abril } & Sem 13 & 4.05 & 5.95 \\
\hline & Sem 14 & 4.18 & 5.45 \\
\hline & Sem 15 & 3.84 & 6.25 \\
\hline & Sem 16 & 4.67 & 5.82 \\
\hline \multirow{4}{*}{ Mayo } & Sem 17 & 4.12 & 5.06 \\
\hline & Sem 18 & 3.85 & 5.34 \\
\hline & Sem 19 & 4.08 & 5.82 \\
\hline & Sem 20 & 4.63 & 5.19 \\
\hline \multirow{4}{*}{ Junio } & Sem 21 & 4.79 & 5.74 \\
\hline & Sem 22 & 4.90 & 5.22 \\
\hline & Sem 23 & 4.83 & 5.97 \\
\hline & Sem 24 & 4.77 & 5.12 \\
\hline \multirow{4}{*}{ Julio } & Sem 25 & 4.10 & 5.36 \\
\hline & Sem 26 & 4.58 & 5.98 \\
\hline & Sem 27 & 4.27 & 6.12 \\
\hline & Sem 28 & 4.19 & 5.69 \\
\hline Promedio & & 4.37 & 5.58 \\
\hline
\end{tabular}

Fuente: Elaboración propia. 


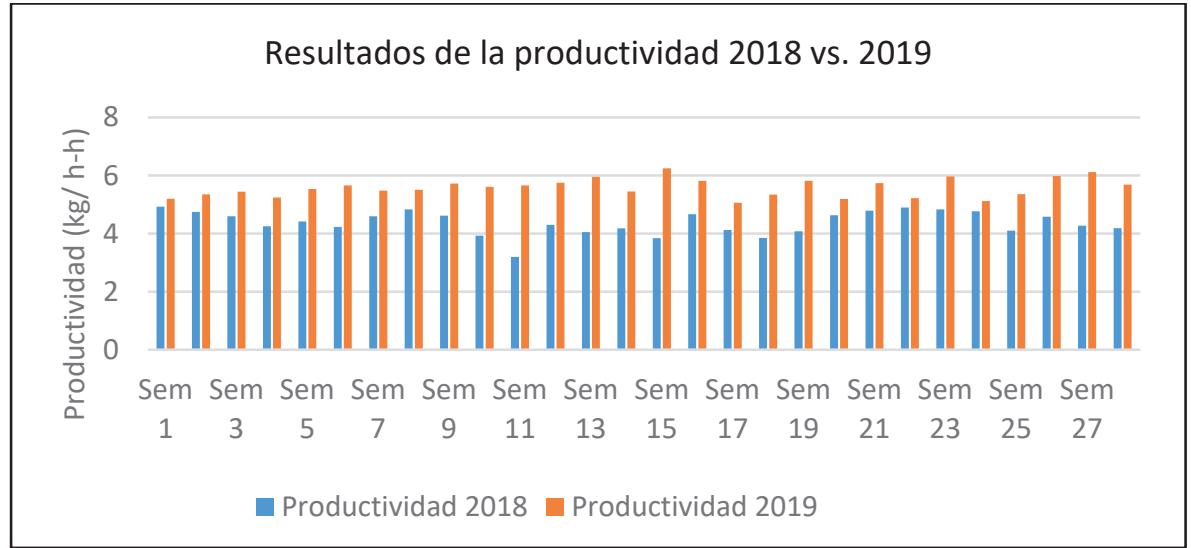

Figura 6. Resultados de la productividad 2018 y 2019. Fuente: Elaboración propia.

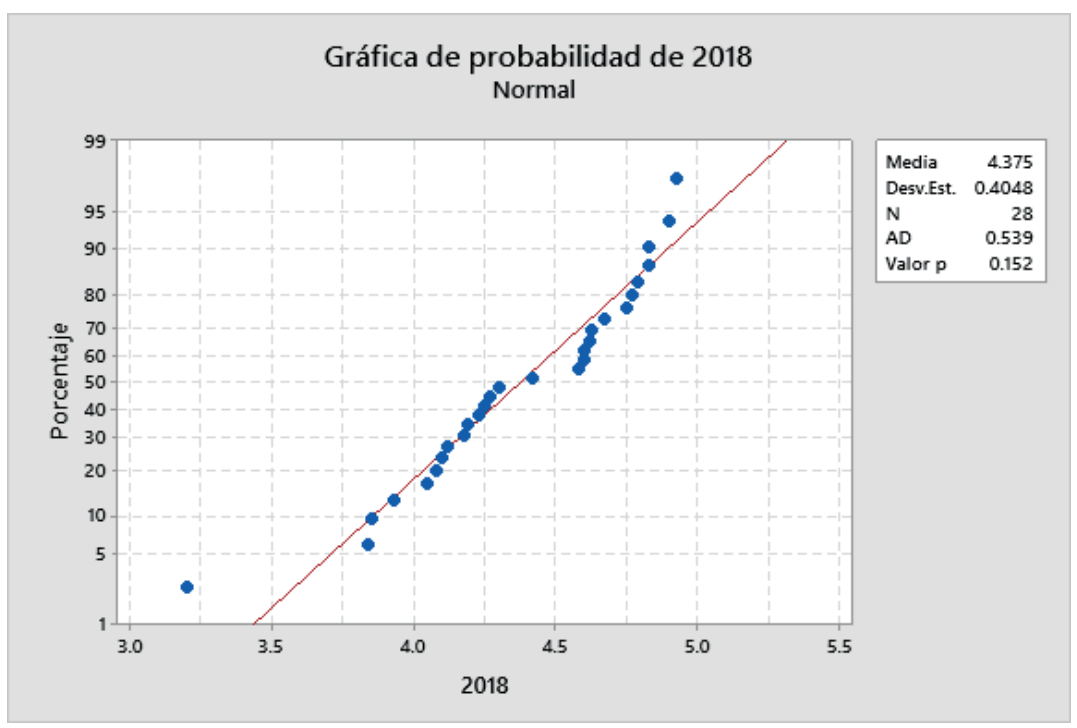

Figura 7. Prueba de normalidad del resultado de la productividad del 2018. Fuente: Elaboración propia.

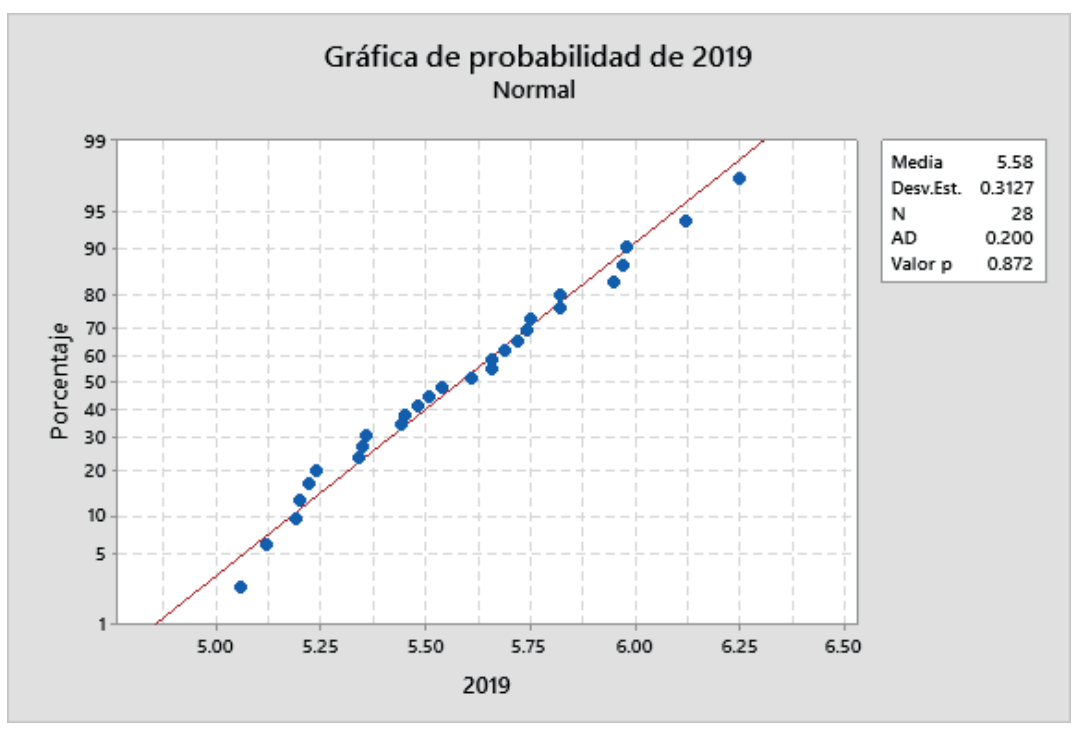

Figura 8. Prueba de normalidad del resultado de la productividad del 2019. Fuente: Elaboración propia. 
Tabla 6. Resultados de la prueba t de Student, productividad del 2018 y 2019.

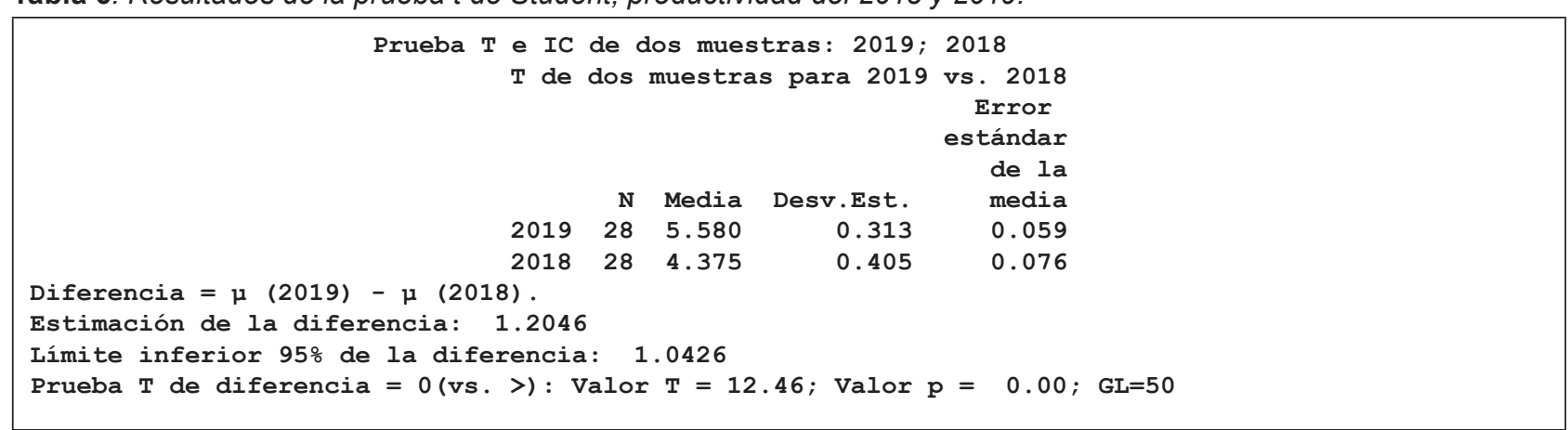

Fuente: Elaboración propia.

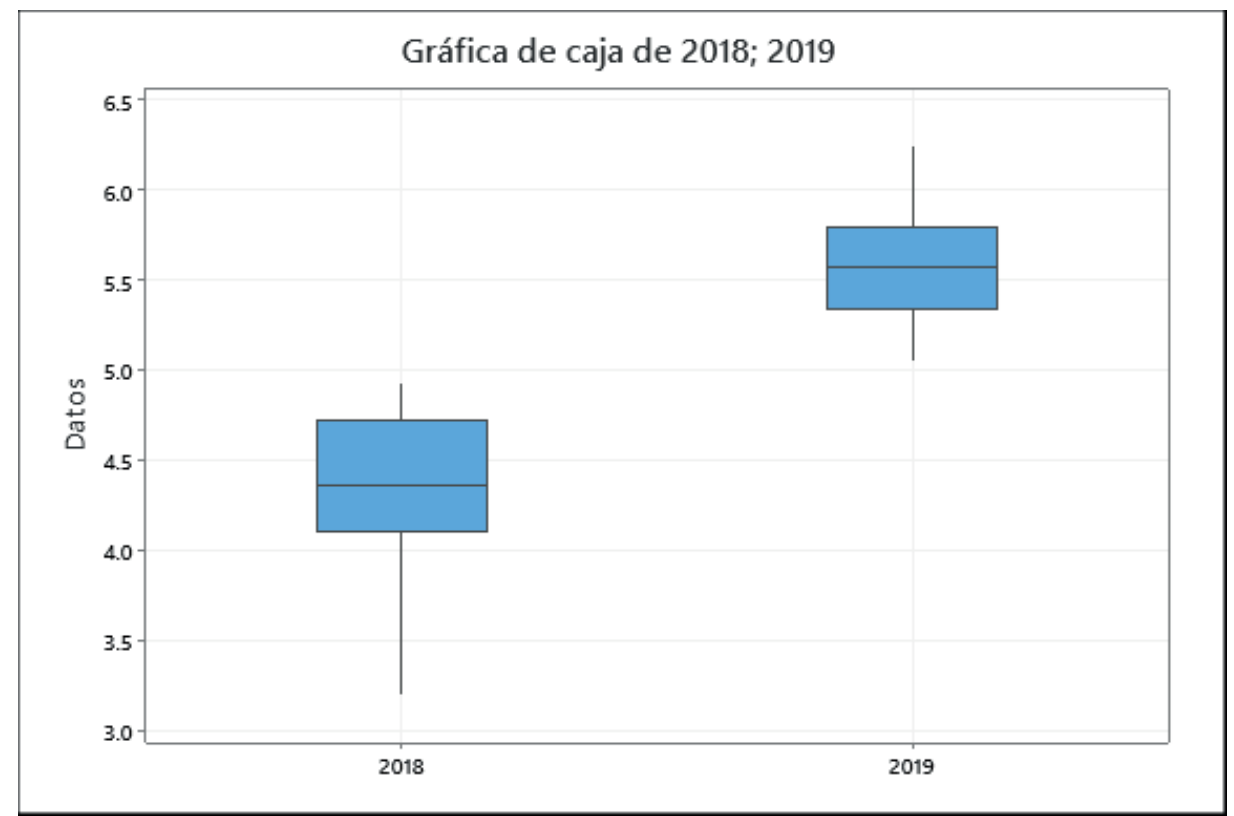

Figura 9. Evaluación de normalidad de los datos obtenidos de la productividad del 2018. Fuente: Elaboración propia.

Los resultados de la aplicación del Lean Manufacturing se pueden generalizar en la mejora de la productividad, el índice de ventas, el incremento de la rentabilidad y la satisfacción de los clientes, empleados y el clima laboral en la empresa manufacturera en estudio.

\section{AGRADECIMIENTO}

A la empresa industrial de fabricación de adhesivos acuosos por brindarme la oportunidad de ser una de las gestoras de dicha implementación y al equipo de trabajo por su compromiso y perseverancia con el cumplimiento de los objetivos como el logro de resultados.

\section{REFERENCIAS BIBLIOGRÁFICAS}

[1] Aldavert, J., Vidal, E., y Lorente, J., y Adalvert, X. (2017). Guía Práctica 5 S para la Mejora Continua. La base del Lean. España: Alda Talent.

[2] Atehortua, Y. (2010). Estudio y aplicación del Kaizen. (Tesis de grado). Universidad Tecnológica de Pereira, Pereira.

[3] Barcia, K., y Hidalgo, D. (2013). Implementación de una Metodología con la Técnica 5S para Mejorar el Área de Matricería de una Empresa Extrusora de Aluminio. Revista Tecnológica ESPOL, 18(1), 69-75. Recuperado de http:// 
www.rte.espol.edu.ec/index.php/tecnologica/ article/view/226/

[4] Bilurbina, L., y Liesa, F. (1990). Materiales no metálicos resistentes a la corrosión. Barcelona, España: Marcombo S.A.

[5] Fernández, R., y Sánchez, C. (1997). Eficacia Organizacional. Concepto, desarrollo y evaluación. Madrid, España: Ediciones Díaz de Santos

[6] Gil, M., Monzon, J., Celma, M., y Giner, F. (1996). Cómo crear y hacer funcionar una empresa: casos prácticos. Madrid, España: ESIC Editorial.

[7] González, F. (2014). Materiales y servicios en reparación de calzado y marroquinería. Madrid, España: IC Editorial.

[8] Huertas, G., y Domínguez, G. (2015). Decisiones estratégicas para la dirección de operaciones en empresas de servicios y turísticas. Barcelona, España: Publicacions i Edicions de la Universitat de Barcelona.

[9] Ishikawa, K. (1997). ¿Qué es el control total de calidad? Bogotá, Colombia: Editorial Norma.
[10] Rajadell, M., y Sánchez, J. (2010). Lean Manufacturing, la evidencia de una necesidad. Madrid, España: Ediciones Díaz de Santos.

[11] Rodríguez, C. (1999). El nuevo escenario. La cultura de calidad y productividad en las empresas. Jalisco, México: ITESO.

[12] Socconini, L. (2018). Lean Manufacturing. Paso a Paso. Barcelona - España: Marge Books.

[13] Vargas, R. (2004). Manual de Implementación Programa 5S. Santander, Colombia: Corporación Autónoma.

[14] Vinodh, S. y Dino, J. (2012). Structural Equation Modelling of lean manufacturing practices. International Journal of Production Research, 50(6), 1598-1607. Recuperado de https://doi.or $\mathrm{g} / 10.1080 / 00207543.2011 .560203$

[15] Womack J., y Jones, D. (1996). Lean Thinking. Barcelona, España: Gestión 2000. 


\section{Application of Lean Manufacturing ( $5 \mathrm{~s}$ and Kaizen) to Increase the Productivity in the Aqueous Adhesives Production Area of a Manufacturing Company}

Edith LuZ VARgas CRISÓSTOMO ${ }^{1}$ José William CAMERo JimÉNEZ ${ }^{2}$

\begin{abstract}
This research was conducted in a manufacturing company that has been experiencing a low-productivity problem for the last four years in the production area of aqueous adhesives, whose productivity values are lower than the expected value $(5 \mathrm{~kg} / \mathrm{mh})$. Accordingly, a strategy for improvement based on the Lean Manufacturing methodology was considered necessary in this study, so the Kaizen methodology and the $5 \mathrm{~S}$ were selected. This was carried out in stages that consisted, first, of a situational diagnosis, followed by the design, implementation and evaluation of the results. These stages were executed in 7 months, from January to July 2019. Upon application of the Lean Manufacturing methodology, the productivity results were evaluated and an average value of $5.58 \mathrm{~kg} / \mathrm{mh}$ was obtained. It is worth mentioning that in 2018 , before the application of Lean Manufacturing, the average productivity value was 4.37 $\mathrm{kg} / \mathrm{mh}$.
\end{abstract}

Keywords: Lean Manufacturing; productivity; $5 S$ and Kaizen.

\section{INTRODUCTION}

Aqueous adhesives are widely used as additives or intermediate products in different industries, such as the wood, paper, and bottling industries, among others. The emergence of new companies in this field is the result of an increasingly competitive market. Therefore, companies need to apply strategies that help them remain competitive and position themselves in the national market in order to survive as an industry, where not only quality is a determining factor, but also the improvement of the production system by eliminating activities that do not add value to the process or to the customer.

The manufacturing company under study, which manufactures aqueous adhesives, has to achieve certain objectives and goals, in addition to making the right decisions to ensure the company's permanence in the market and a high profitability that will result in benefits for its workers and shareholders; however, productivity has been low in the last 4 years. New methodologies based on process improvement, such as Lean Manufacturing, which increases productivity by eliminating waste, must be applied to reverse this situation.

The Lean Manufacturing methodology is defined as a management model of excellence and continuous improvement that focuses on the elimination of waste that does not add value to the product and is comprised of different tools. $5 S$ and Kaizen tools were applied in this research, for which a literature review of Lean Manufacturing was undertaken, followed by the design, implementation and evaluation of the results with statistical methods.

The Lean philosophy is considered innovative and relevant within companies, since it allows optimizing production processes by eliminating activities that do not add value in the different stages of operations and that do not provide benefits to the company.

1 Chemical Engineer from Universidad Nacional del Centro del Perú (Junin, Peru). Currently working as an independent consultant (Lima, Peru). ORCID: https://orcid.org/0000-0001-9637-5365 Corresponding author: edith luz91@hotmail.com

2 Degree in Statistics from Universidad Nacional de Ingeniería (Lima, Peru). Currently teaches at the Escuela Profesional de Estadística of Universidad Nacional de Ingeniería (Lima, Peru). ORCID: https://orcid.org/0000-0003-0517-3237 E-mail: jcameroj@uni.edu.pe 
This elimination is applied from the start to the end of production, which improves economy and saves resources.

Optimizing processes and improving productivity by reducing downtime translates into increased profits, improved product quality and a culture of change in people's thinking, in order to achieve efficiency in the production process, that is, to produce more with fewer resources and less effort.

All production and service processes must be evaluated in order to generalize the results obtained to other organizations and identify where this methodology needs to be applied, essentially to provide solutions to problems through continuous improvement. Lean Manufacturing is implemented through six steps: formation of a Lean implementation team, training, diagnosis (identification of waste), Lean methodology application, analysis of results and elaboration of action plans focused on the reduction of process time to achieve cost reduction, defect reduction, improvement of the organization's profitability, among others.

Our general objective is "Apply Lean Manufacturing (5S and Kaizen) to increase productivity in aqueous adhesives production area of a manufacturing company". Based on this, the specific objectives are "Analyze the current productivity situation of the aqueous adhesives production area of a manufacturing company", "Apply the Lean Manufacturing methodology in the aqueous adhesives production area of a manufacturing company" and "Evaluate the productivity after applying the Lean Manufacturing methodology in the aqueous adhesives production area of a manufacturing company".

\section{JUSTIFICATION}

The adhesives manufacturing sector is growing and is expected to make significant progress in the coming years, as it offers benefits such as equivalent and sometimes better performance than other products used in assembly and surface sealing, with advantages in terms of cost reduction. Adhesives are also used as intermediate products and inputs in a variety of industrial products, such as labeling adhesive, laminating adhesive (toilet paper), cardboard adhesive, among others; therefore, aqueous adhesives production has become an attractive business.

At present, the number of aqueous adhesive manufacturing companies offering quality products and services has increased, resulting in a competitive market. Therefore, this research focuses on applying an improvement strategy through Lean Manufacturing (5S and KAIZEN) and evaluating the impact on productivity growth.

\section{HYPOTHESISIS}

- $\mathrm{H}_{0}=$ Productivity in 2019 is equal to productivity in 2018.

- $\mathrm{H}_{\mathrm{a}}=$ Productivity in 2019 is higher than productivity in 2018.

\section{THEORETICAL FRAMEWORK}

\section{Adhesives}

Adhesive is defined as the application of all the forces that oppose the division of molecules of different contexts; therefore, adhesion is the resistance that opposes the separation of bodies (Bilurbina \& Liesa, 1990).

\section{Aqueous Adhesives}

Water-based adhesives are solutions of polymers (of vegetable or synthetic nature) dissolved in water and are characterized by having a slower setting speed than solvent-based adhesives, since water takes longer to evaporate than organic adhesives (Gonzáles, 2014).

Water-based adhesives are one of the best alternatives to those using organic solvents, which are much more toxic and polluting (Gonzáles, 2014).

\section{Lean Manufacturing}

Lean Manufacturing is understood as the search for the improvement of the manufacturing system through the elimination or reduction of waste or muda, consisting of all actions that do not add value to the product or service produced by activities that consume resources without creating any value and for which customers are not willing to pay (Womack \& Jones, 1996).

The Lean Manufacturing methodology aims to minimize waste on an ongoing basis to maximize process steps, focusing on cost reduction and minimization of waste and materials in the supply chain (Vinodh \& Dino, 2012). This can be summarized in the following objectives:

- Leverage the use of organizational assets;

- Improve external and internal customer satisfaction; 
- Eliminate waste (activities that do not add value) in the manufacturing process.

\section{The Pillars of Lean Manufacturing}

\section{- Total Quality Control}

Total quality control is quality assurance from the earliest stages of product development. It is designed to ensure that products or services meet quality requirements, which is accomplished involving all employees in all processes, thus giving rise to organizationwide quality control, considered to be the most important quality of Japanese quality control (Ishikawa, 1997).

Quality control is applied to an organization mainly to know the specifications set by the product engineering and to provide assistance to the manufacturing department, so that the production reaches these specifications. As such, it is concerned with the collection and analysis of large amounts of data that are then presented to different departments for subsequent evaluation.

\section{- Continuous Improvement}

Continuous improvement is the permanent goal of organizations, as it focuses on problem solving. The PDCA cycle, today considered an essential improvement tool, was introduced by Deming, who described it in 4 steps (Socconini, 2018):

- Plan: A change or test aiming at improvement.

- Do: Test the change or test, preferably, on a small scale.

- Check: Review the results - what have you learned?

- Act: Adopt the change, abandon it, or go through the cycle again, possibly under different environmental conditions.

\section{- Just-In-Time (JIT)}

Taiichi Ohno, executive vice president of Toyota Motor Corporation, developed the just-in-time production system seeking cost reduction through the elimination of waste. Based on concepts developed by Henry Ford and Walter Shewhart in the 1920s and 1930s, Ohno developed a philosophy of manufacturing excellence that surpassed all previous ones (Rajadell \& Sánchez, 2010).

\section{- Productivity}

Productivity is the correlation between production and the optimal use of financial, material and human resources to achieve organizational objectives, increase the quality of services and goods produced a pursue the development of employees (Rodríguez, 1999).

\section{- Effectiveness}

Effectiveness is the ability of an organization to increase profits by different means, including the capacity to achieve the desired effect and the management of the inputs and outputs of the environment, such as politics and others (Fernández \& Sánchez, 1997).

\section{- Efficiency}

Efficiency is achieved when the objectives set are attained with the minimum amount of inputs. For instance, we can increase efficiency in a workday by eliminating breaks and reducing lunch time, which would increase production; however, it is possible that the number of errors will also increase due to employee fatigue, i.e., an efficient production system of inefficient products will have been achieved. Therefore, it is proposed that productivity is a relationship between effectiveness and efficiency achieved, monitoring manufacturing costs in which effectiveness is doing the right thing and efficiency is the way of doing things well (Huertas \& Domínguez, 2015).

\section{- The $5 \mathrm{~S}$ :}

The $5 \mathrm{~S}$ are a methodology for improvement that originated in Japan. It consists of sorting, order and cleanliness of the area, standardization of processes and personnel discipline, so that they become habits in the work areas and a culture of labor improvement is achieved. This method can be applied to any field and in all work areas of a company (Barcia \& Hidalgo, 2013)

o Seiri: Sort. Determining and differentiating all that is really necessary or essential for our work area. Essential elements are kept, to later classify them while disposing of the unnecessary. Thus reducing elements at the workplace, and keeping only those essential to complete the processes and tasks with maximum effectiveness and 
efficiency (Aldavert, Vidal, \& Lorente, 2017).

o Seiton: Set in order. Placing what is necessary in an easily accessible place, placing useful things in order according to Safety/Quality/Efficiency criteria.

- Safety: They should not fall, they should not move, they should not be in the way.

- Quality: That They do not get hit, rust, mix or deteriorate.

- Efficiency: Minimize lost time (Vargas, 2004).

o Seiso: Shining. It intends to encourage the attitude of cleanliness of the work place and maintaining the classification and order of the elements. It should be supported by a strong training program and the supply of the necessary elements for its implementation, as well as the time required for its execution (Vargas, 2004).

o Seiketsu: Standardize. Constantly maintaining the order, cleanliness and hygiene of the work area. It is achieved by cleaning regularly, keeping everything in its place and in order, establishing procedures and plans to maintain order and cleanliness (Vargas, 2004). o Shitsuke: Sustain. Getting into the habit of applying the 5 S's in the work area, respecting and enforcing the rules of the work area, always using protective implements and maintaining cleanliness, so that they become daily habits (Vargas, 2004).

\section{- Kaizen}

The word Kaizen means "to improve". When applied in organizations as a continuous improvement in the way of doing things, it generates an impact in the work place, as well as in the personal, family and social environment.

A culture of continuous improvement, which includes all workers, is a complementary effort towards performance improvement at all levels of the organization, directed towards the satisfaction of cross-functional goals such as quality, costs and human potential, in order to achieve greater customer satisfaction (Gil, Monzon, Celma, \& Giner 1996).

There are 10 basic principles in the application of Kaizen, which resulted from various experiences in different Japanese companies. It became known that training and competence development of employees should encourage Kaizen culture development, where principles should be internalized and applied quite naturally.

According to Atehortua (2010), the Kaizen principles are:

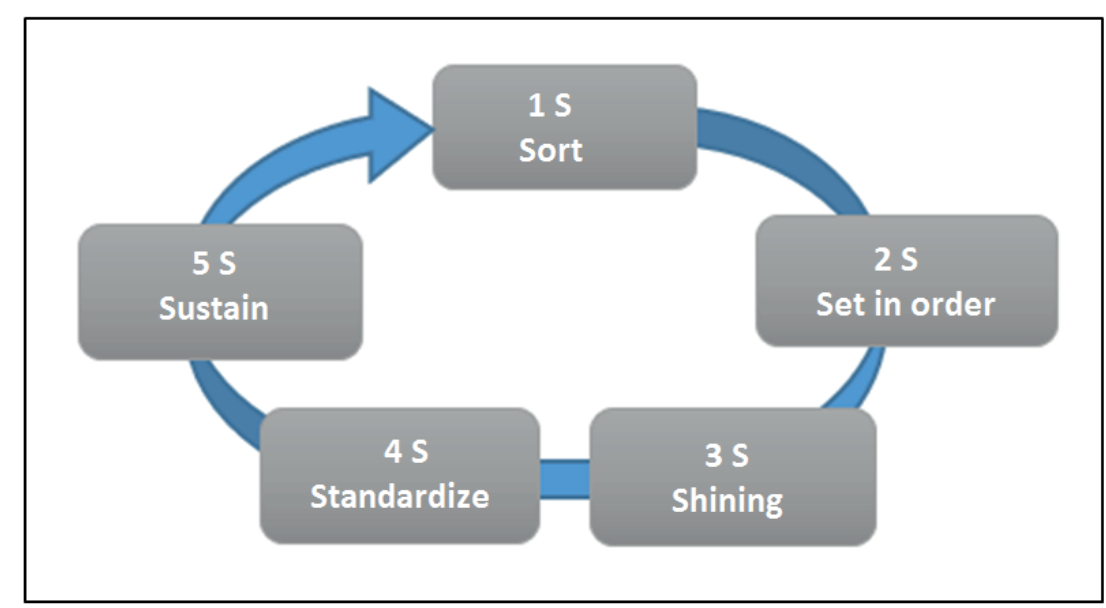

Figure 1. The 5S Lean practices. Source: Prepared by the authors. 
1. Focus on customer requirements.

2. Constantly make small improvements.

3. Openly identify, recognize and address problems.

4. Encourage open communication.

5. Form "Kaizen" work teams.

6. Conduct Kaizen projects involving multi-functional individuals.

7. Maintain good working relationships.

8. Encourage self-discipline by means of recognition.

9. Maintain communication with employees.

10. Promote the development of competencies in all employees.

\section{METHODOLOGY}

\section{Productivity Analysis}

The values of the last 5 years are used for the productivity analysis (Table 1). Only in 2014, productivity exceeded the value of $5.00 \mathrm{~kg} / \mathrm{mh}$, which is the value expected by the manufacturing company.

Table 1. Productivity Results by Year.

\begin{tabular}{|c|c|}
\hline Year & $\begin{array}{c}\text { Productivity } \\
\mathbf{k g} / \mathbf{m h}\end{array}$ \\
\hline 2014 & 5.23 \\
2015 & 4.75 \\
2016 & 4.60 \\
2017 & 4.87 \\
2018 & 4.37 \\
\hline
\end{tabular}

Source: Prepared by the authors.

\section{Productivity Determination}

The following formula is used to determine productitvity in the company:

$$
\text { Productivity }=\frac{\text { Production of the month }(\mathrm{Kg})}{\text { Total man }- \text { hours }(\mathrm{mh})}
$$

\footnotetext{
Production in the aqueous adhesives manufacturing plant
} $=\overline{\text { Production personnel }+ \text { administrative production personnel }}$

The methodology for the application of Lean Manufacturing $5 \mathrm{~S}$ and Kaizen is shown in Figure 2.

\section{Selection of the Workteam}

Production personnel and personnel from the continuous improvement area are selected to conduct and support the activities for the application of Lean Manufacturing tools (5S and Kaizen).

\section{Training}

The team selected is trained in Lean Manufacturing and the stages of its application; instructors from the Lean Six Sigma Institute of Mexico are in charge of training.

\section{Assessment of the 5 S's}

An assessment of the current situation of the 5 S's in the aqueous adhesives production area was carried out using a check list format to process the data. The images of the current situation are shown in Figure 3.

\section{Application of the $5 \mathrm{~S}$}

The application consisted of following the guidelines of the $5 \mathrm{~S}$ Implementation Handbook, provided by the Continuous Improvement area, which is summarized in the following steps:

a. Lay out elaboration (5S drawing). Delimitation of the zones or areas where the $5 S$ are to be implemented.

b. Regarding 1S: A list of necessary items is created and unnecessary items are removed.

c. Regarding 2S: All the necessary items, including equipment, machines, furniture, tools, materials, among others, are located and labeled.

d. Regarding 3S: All necessary items are kept clean, a cleaning plan is implemented and compliance is monitored.

e. Regarding 4S: It is verified that labels and markings comply with the standardization rules set forth in the $5 \mathrm{~S}$ Implementation Handbook.

f. Regarding 5S: $5 S$ audits are carried out and a person responsible within the area is designated to address issues.

\section{Application of Kaizen}

Changes are made to improve the production process of the best-selling product through the implementation of a Kaizen. The implementation comprises the following stages. 


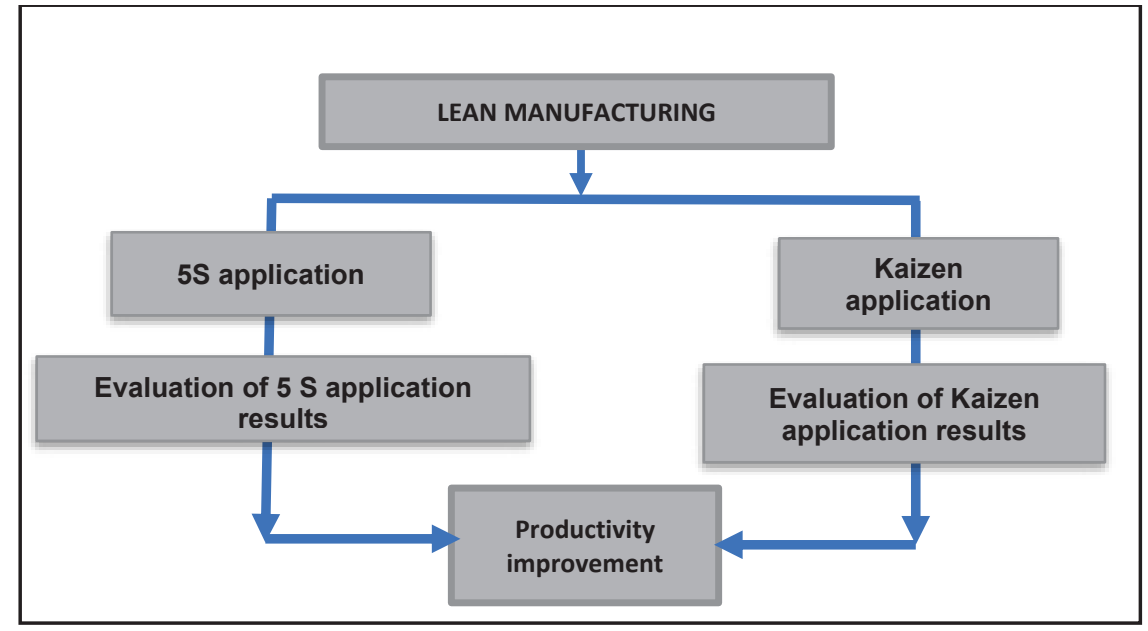

Figure 2. Implementation of Lean Manufacturing. Source: Prepared by the authors.

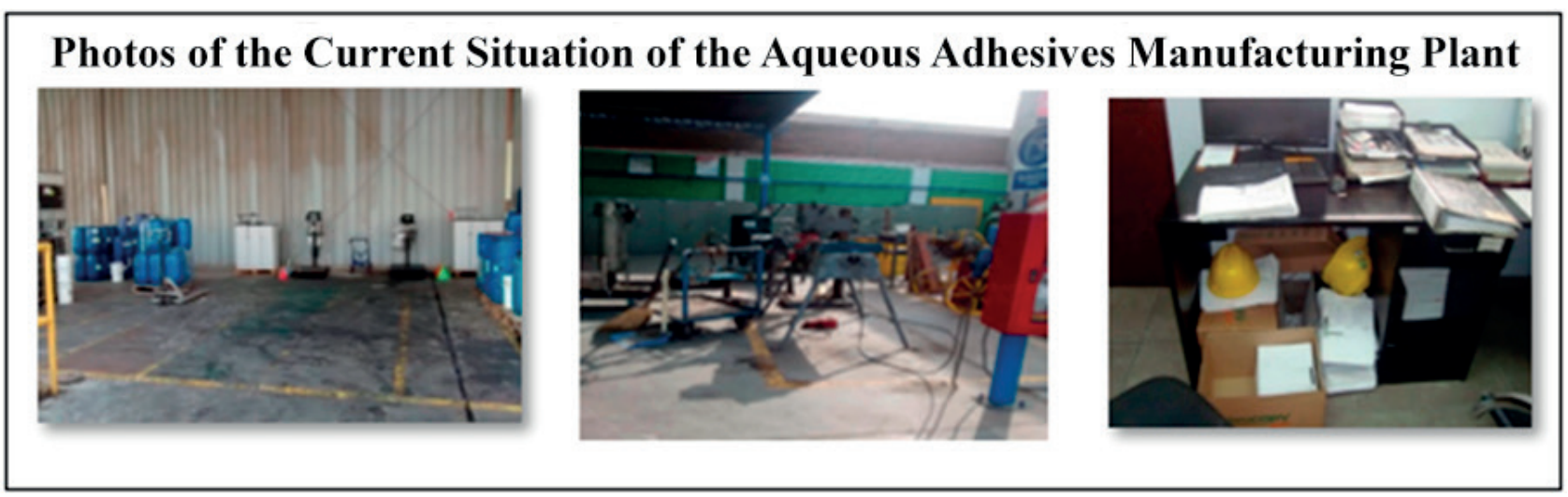

Figure 3. Current situation of the aqueous adhesives manufacturing plant.

Source: Prepared by the authors.

a. The problem in the production process of aqueous adhesives is identified, which is the excessive manufacturing process time of the best-selling product.

b. The manufacturing process of the product is described, consisting of 10 stages in the production process.

c. A cause analysis is performed using the brainstorming and Pareto techniques. The root cause is the excessive cooling time of the reactor used in the manufacturing process due to lack of maintenance ( excessive scale build-up).

d. An action plan, including maintenance activities, is drafted in collaboration with the maintenance area of the equipment used in the production process.

e. The implemented activities are followed up and their effectiveness is evaluated.

\section{Results of the $5 \mathrm{~S}$}

a. The results of the 5S diagnosis, carried out before the start of the Lean Manufacturing application in January 2018, are shown in Table 2 and in the images in Figure 4.

b. The results of the $5 \mathrm{~S}$ audit, conducted after applying the Lean Manufacturing methodology, in the months of April to July 2019, are shown in Table 3 and in Figure 5.

\section{Results Obtained from Kaizen}

Table 4 shows the results of applying Kaizen to address the problem encountered (excessive manufacturing time of the product with the highest sales turnover); the application of Kaizen reduced manufacturing times by up to 2 hours and 23 minutes (Table 4). 
Table 2. Results of the 5S Assessment.

\begin{tabular}{|l|l|c|}
\hline \multicolumn{2}{|c|}{ Pillars of 5 S } & Assessment of 5 S \\
\hline 1 S & Sort & 3.0 \\
\hline 2 S & Set in order & 2.5 \\
\hline 3 S & Shining & 2.7 \\
\hline 4 S & Standardize & 2.2 \\
\hline 5 S & Sustain & 3.5 \\
\hline Average & 2.8 \\
\hline
\end{tabular}

Source: Prepared by the authors.

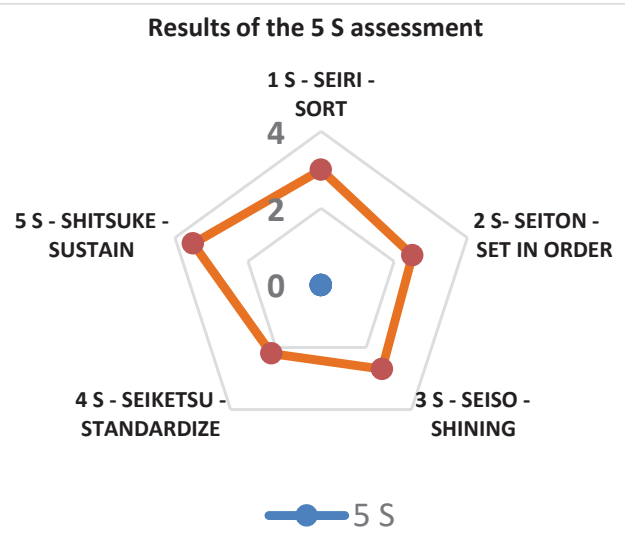

Figure 4. Results of the $5 \mathrm{~S}$ assessment. Source: Prepared by the authors.

Table 3. Results of the 5 S Audit.

\begin{tabular}{|c|c|c|}
\hline \multicolumn{2}{|c|}{ Months (2019) } & 5 S Audit Score \\
\hline 1 & April & 4.0 \\
\hline 2 & May & 4.0 \\
\hline 3 & June & 4.5 \\
\hline 4 & July & 4.5 \\
\hline
\end{tabular}

Source: Prepared by the authors.

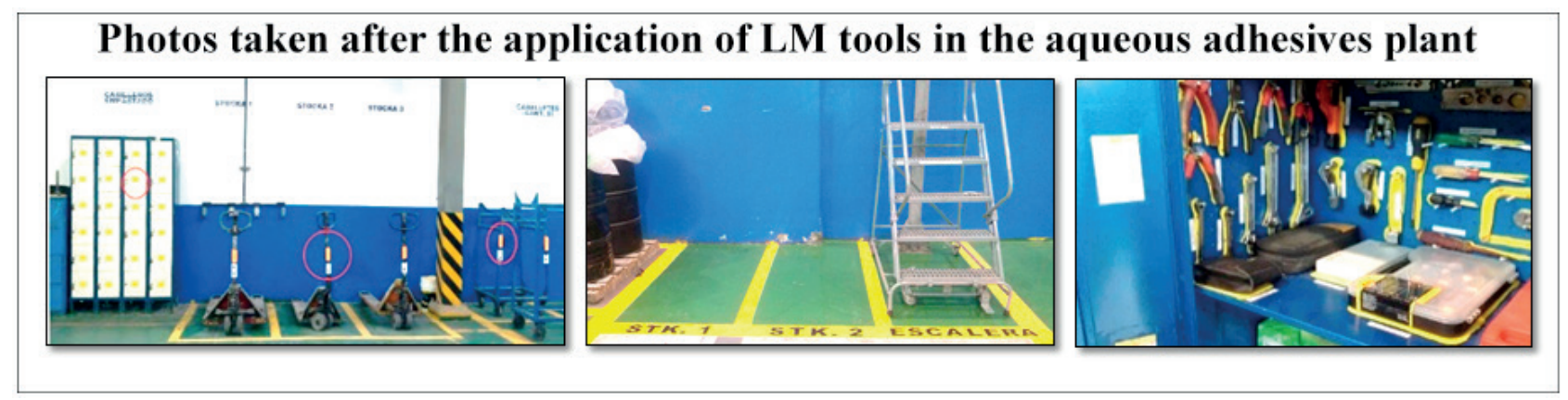

Figure 5. Results of the $5 \mathrm{~S}$ assessment.

Source: Prepared by the authors.

\section{Productivity Data}

Table 5 shows the productivity data obtained in the aqueous adhesives production area for 2018 (before applying the Lean Manufacturing methodology) and the productivity data for 2019 (after applying the Lean Manufacturing methodology) (Figure 6).

\section{HYPOTHESIS TESTING}

Pursuant to the hypothesis statement and the data shown in Table 5, Minitab software is used to evaluate the null hypothesis at a significance level of $\alpha$ $=0.05$; Student's t-test and the box plot are used to 
Table 4. Results of Applying Kaizen.

\begin{tabular}{|l|c|c|}
\hline \multirow{2}{*}{ Kaizen Tools } & $\begin{array}{c}\text { Months } \\
\text { (2019) }\end{array}$ & $\begin{array}{c}\text { Manufacturing Time } \\
\text { (hours) }\end{array}$ \\
\hline \multirow{3}{*}{ Before applying Kaizen } & January & $20: 15: 00$ \\
\cline { 2 - 3 } & February & $20: 04: 00$ \\
\cline { 2 - 3 } & March & $20: 10: 00$ \\
\hline \multirow{3}{*}{ After applying Kaizen } & April & $18: 19: 00$ \\
\cline { 2 - 3 } & May & $18: 46: 00$ \\
\cline { 2 - 3 } & June & $17: 26: 00$ \\
\cline { 2 - 3 } & July & $17: 09: 00$ \\
\hline
\end{tabular}

Source: Prepared by the authors.

Table 5. Productivity Data for 2018 and 2019.

\begin{tabular}{|c|c|c|c|}
\hline Month & Week & $\begin{array}{c}\text { Productivity } 2018 \\
(\mathrm{~kg} / \mathrm{mh})\end{array}$ & $\begin{array}{c}\text { Productivity } 2019 \\
(\mathrm{~kg} / \mathrm{mh})\end{array}$ \\
\hline \multirow{4}{*}{ January } & Week 1 & 4.93 & 5.20 \\
\hline & Week 2 & 4.75 & 5.35 \\
\hline & Week 3 & 4.60 & 5.44 \\
\hline & Week 4 & 4.25 & 5.24 \\
\hline \multirow{4}{*}{ February } & Week 5 & 4.42 & 5.54 \\
\hline & Week 6 & 4.23 & 5.66 \\
\hline & Week 7 & 4.60 & 5.48 \\
\hline & Week 8 & 4.83 & 5.51 \\
\hline \multirow{4}{*}{ March } & Week 9 & 4.62 & 5.72 \\
\hline & Week 10 & 3.93 & 5.61 \\
\hline & Week 11 & 3.20 & 5.66 \\
\hline & Week 12 & 4.30 & 5.75 \\
\hline \multirow{4}{*}{ April } & Week 13 & 4.05 & 5.95 \\
\hline & Week 14 & 4.18 & 5.45 \\
\hline & Week 15 & 3.84 & 6.25 \\
\hline & Week 16 & 4.67 & 5.82 \\
\hline \multirow{4}{*}{ May } & Week 17 & 4.12 & 5.06 \\
\hline & Week 18 & 3.85 & 5.34 \\
\hline & Week 19 & 4.08 & 5.82 \\
\hline & Week 20 & 4.63 & 5.19 \\
\hline \multirow{4}{*}{ June } & Week 21 & 4.79 & 5.74 \\
\hline & Week 22 & 4.90 & 5.22 \\
\hline & Week 23 & 4.83 & 5.97 \\
\hline & Week 24 & 4.77 & 5.12 \\
\hline \multirow{4}{*}{ July } & Week 25 & 4.10 & 5.36 \\
\hline & Week 26 & 4.58 & 5.98 \\
\hline & Week 27 & 4.27 & 6.12 \\
\hline & Week 28 & 4.19 & 5.69 \\
\hline Average & & 4.37 & 5.58 \\
\hline
\end{tabular}

Source: Prepared by the authors. 
demonstrate whether the null hypothesis is accepted or rejected.

\section{Statistical Normality Test}

The Anderson Darling test is used for the statistical test of normality for the 2018 and 2019 productivity data. (Figure 7 and 8). The data were accepted as they had a $p$-value $>\alpha$ at $95 \%$ confidence.

\section{Student's t-Test}

After determining that the data have a normal distribution, Student's t-test is performed for both samples. Table 6 shows the result, where a $p$-value $<\alpha$ $(0.00<0.05)$ was obtained; therefore, the null hypothesis is rejected and the alternative hypothesis is accepted. Consequently, productivity in 2019 is higher than productivity in 2018 due to the implementation of the Lean Manufacturing methodology.

\section{Box Plot}

The increase in productivity can be observed in the box plot in Figure 9.

\section{DISCUSSION}

Upon applying the Lean Manufacturing methodologies (5S and Kaizen), productivity results of the aqueous adhesives production area of a manufacturing company were improved. These results were corroborated via hypothesis testing. It was thus

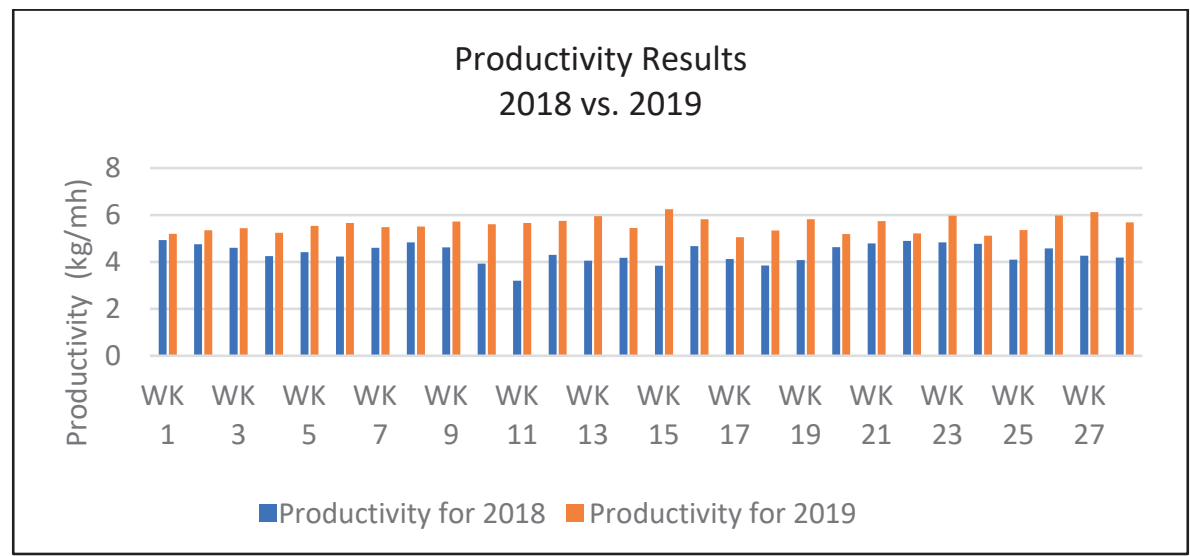

Figure 6. Productivity results for 2018 and 2019. Source: Prepared by the authors.

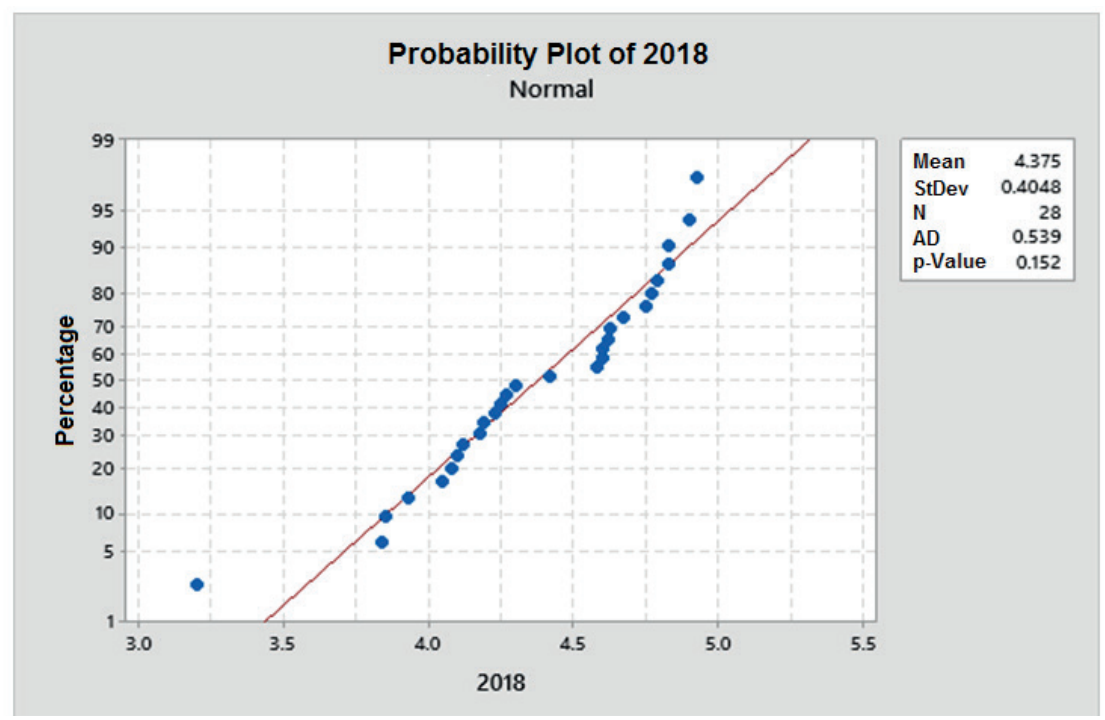

Figure 7. Normality test of the 2018 productivity result. Source: Prepared by the authors. 


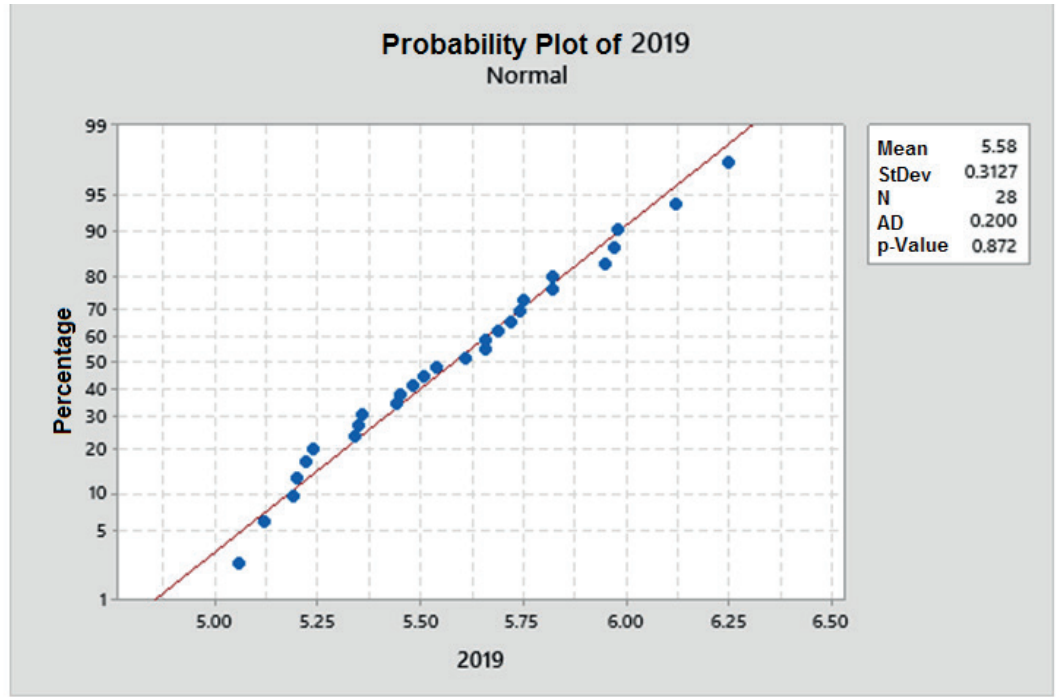

Figure 8. Normality test of the 2019 productivity result. Source: Prepared by the authors.

Table 6. Results of Student's t-Test, Productivity of 2018 and 2019.

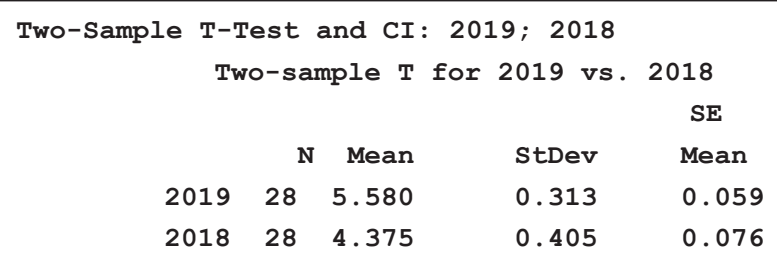

Fuente: Elaboración propia.

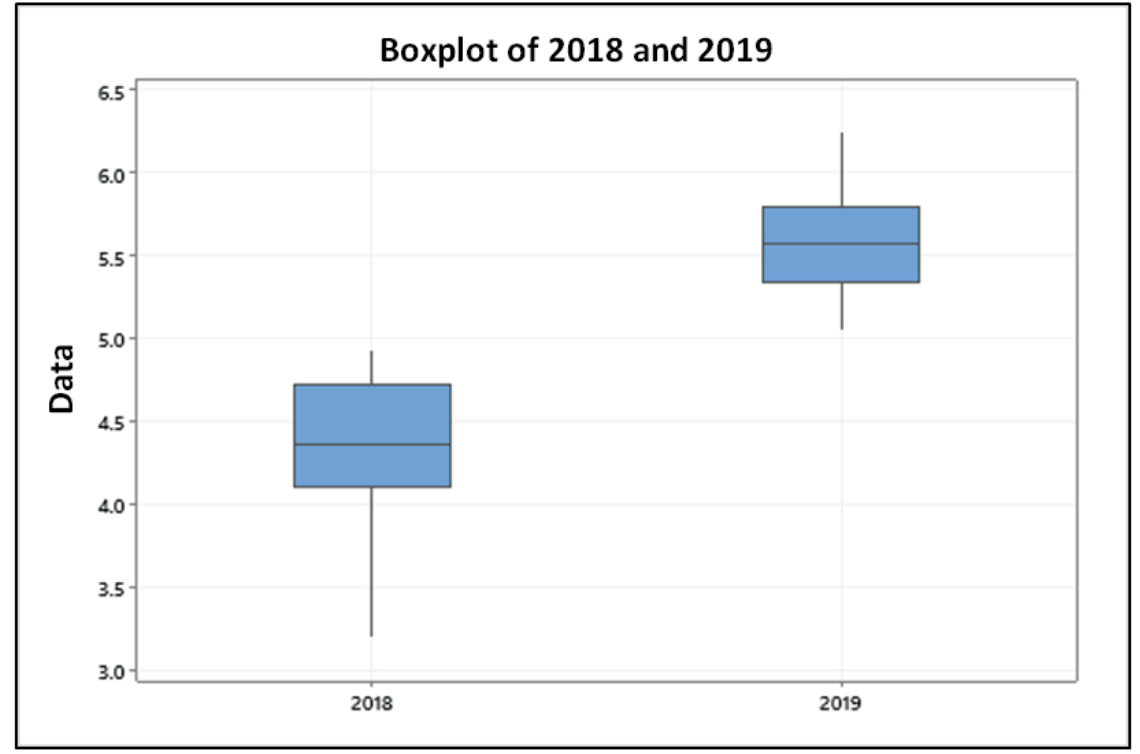

Figure 9. Normality testing of the 218 productivity data. Source: Prepared by the authors. 
demonstrated that productivity increases upon applying Lean Manufacturing.

\section{CONCLUSIONS}

A baseline or diagnostic study of $5 \mathrm{~S}$ was carried out before the application of Lean Manufacturing, obtaining an initial average value of 2.8. At the end of the $5 \mathrm{~S}$ application, an average value of 4.03 was obtained as an audit result by reducing unnecessary time spent searching for materials and transporting personnel, in addition to improving the organization and cleanliness of the aqueous adhesives production area.

By applying the Kaizen methodology, the manufacturing process time of the product with the highest sales rate in the aqueous adhesives production area was reduced. Before applying Kaizen, the manufacturing time was 20:15 hours and, after the application, a manufacturing time of 17:09 hours was obtained, i.e., the manufacturing time was reduced by 3 hours and 6 minutes.

The results of the application of Lean Manufacturing can be used to improve productivity, sales rate, profitability, customer satisfaction, employees' satisfaction and work environment in the manufacturing company under study.

\section{ACKNOWLEDGMENT}

To the industrial company that manufactures aqueous products for giving me the opportunity to participate in the implementation of this methodology. I would also like to thank the work team for their commitment and perseverance in meeting the objectives and achieving results.

\section{REFERENCES}

[1] Aldavert, J., Vidal, E., \& Lorente, J., \& Adalvert, X. (2017). Guía Práctica 5 S para la Mejora Continua. La base del Lean. Spain: Alda Talent.

[2] Atehortua, Y. (2010). Estudio y aplicación del Kaizen. (Graduate thesis). Universidad Tecnológica de Pereira, Pereira.

[3] Barcia, K., \& Hidalgo, D. (2013). Implementación de una Metodología con la Técnica 5S para Mejorar el Área de Matricería de una Empresa Extrusora de Aluminio. Revista Tecnológica - ESPOL, 18(1), 69-75. Retrieved from http:// www.rte.espol.edu.ec/index.php/tecnologica/ article/view/226/
[4] Bilurbina, L., \& Liesa, F. (1990). Materiales no metálicos resistentes a la corrosión. Barcelona, Spain: Marcombo S.A.

[5] Fernández, R., \& Sánchez, C. (1997). Eficacia Organizacional. Concepto, desarrollo y evaluación. Madrid, Spain: Ediciones Díaz de Santos

[6] Gil, M., Monzon, J., Celma, M., \& Giner, F. (1996). Cómo crear y hacer funcionar una empresa: casos prácticos. Madrid, Spain: ESIC Editorial.

[7] González, F. (2014). Materiales y servicios en reparación de calzado y marroquinería. Madrid, Spain: IC Editorial.

[8] Huertas, G., \& Domínguez, G. (2015). Decisiones estratégicas para la dirección de operaciones en empresas de servicios y turísticas. Barcelona, Spain: Publicacions i Edicions de la Universitat de Barcelona.

[9] Ishikawa, K. (1997). ¿Qué es el control total de calidad? Bogotá, Colombia: Editorial Norma.

[10] Rajadell, M., \& Sánchez, J. (2010). Lean Manufacturing, la evidencia de una necesidad. Madrid, Spain: Ediciones Díaz de Santos.

[11] Rodríguez, C. (1999). El nuevo escenario. La cultura de calidad y productividad en las empresas. Jalisco, Mexico: ITESO.

[12] Socconini, L. (2018). Lean Manufacturing. Paso a Paso. Barcelona - Spain: Marge Books.

[13] Vargas, R. (2004). Manual de Implementación Programa 5S. Santander, Colombia: Corporación Autónoma.

[14] Vinodh, S. \& Dino, J. (2012). Structural Equation Modelling of lean manufacturing practices. International Journal of Production Research, 50(6), 1598-1607. Retrieved from https://doi.or g/10.1080/00207543.2011.560203

[15] Womack J., \& Jones, D. (1996). Lean Thinking. Barcelona, Spain: Gestión 2000. 